\title{
Interaction between acrylic substrates and RAD16-I peptide in its self-assembling
}

M. Arnal-Pastor ${ }^{1}$, Débora González-Mora ${ }^{1, \#}$, Fernando García-Torres ${ }^{1, \text { \#, M. Monleón Pradas }}{ }^{1,2}$, A. Vallés-Lluch ${ }^{1, *}$

${ }^{I}$ Center for Biomaterials and Tissue Engineering, Universitat Politècnica de València, C. de Vera s/n, 46022, Valencia, Spain

${ }^{2}$ Networking Research Center on Bioengineering, Biomaterials and Nanomedicine, Valencia, Spain

*Corresponding author.Tel.: +34963877000 (88936); fax: +34963877276.E-mail: avalles@ter.upv.es

\# Equal contribution

\begin{abstract}
Self-assembling peptides (SAP) are widely used as scaffolds themselves, and recently as fillers of microporous scaffolds, where the former provides a cell-friendly nanoenvironment and the latter improves its mechanical properties. The characterization of the interaction between these short peptides and the scaffold material is crucial to assess the potential of such a combined system. In this work, the interaction between poly(ethyl acrylate) (PEA) and 90/10 ethyl acrylate-acrylic acid copolymer P(EAcoAAc) with the SAP RAD16-I has been followed using a bidimensional simplified model. By means of the techniques of choice (congo red staining, atomic force microscopy (AFM), and contact angle measurements) the interaction and self-assembly of the peptide has proven to be very sensitive to the wettability and electronegativity of the polymeric substrate.
\end{abstract}

Keywords: self-assembling peptide; ethyl acrylate; acrylic acid; substrate interaction; wettability 


\section{Introduction}

Self-assembling peptides (SAPs) are synthetic materials, consisting of short peptides, which under a change in their environment, like a $\mathrm{pH}$ modification or the addition of salts [1], are capable to undergo a self-assembling process. RAD16-I (Ac-RADARADARADARADA-CONH ${ }_{2}$ in particular, adopts beta-sheet configurations that are stable through $\mathrm{pH}$ or temperature variations up to $\mathrm{pH} 11$ and $90^{\circ} \mathrm{C}$ [2]. If the peptide concentration is high enough, and the conditions are adequate, RAD16-I is capable to form a 3D network that mimics the extracellular matrix (ECM), with fibers in the range of 10-20 nm diameter and mesh sizes (pores) about 50-100 $\mathrm{nm}$ in diameter [3].

RAD16-I presents several advantages over animal-derived ECM materials like Matrigel@ or collagen membranes: there is no risk of illness transmission, and all batches are homogeneous [4]. Moreover, RAD16-I is non-immunogenic and produces no inflammatory reactions in animals $[4,5]$. RAD16-I's great potential for regenerative medicine has been tested with different cell lines [6-10], and it has been found to be an interesting vehicle to encapsulate cells or to be used as injectable cell carrier [11]. Despite the good biological performance achieved, the mechanism by which cells attach to the peptide chains is still unclear; so far it has been established that cells do not attach to SAP via RGD-dependent integrin receptors [12]. Interestingly, SAP lack of known cell adhesion sites, therefore they could act instead as templates on which cells can deposit its own matrix [6].

Even after the self-assembly, SAPs are very weak materials, difficult to manipulate, move or implant if is not through injection. Therefore, it might be of interest to combine them with other materials to provide the structural integrity and manipulability that these peptides lack. For instance, SAPs have been used in combination with cellulose tubular membranes [13], to coat the surface of 
chitosan electrospun fibers [14], also as fillers of porous titanium foams [15], or combined with poly (ethyl acrylate), PEA, porous scaffolds [16,17] or (polycaprolactone methacryloyloxyethyl ester) scaffolds [18]. In such approaches, the peptide is intended to enhance the biological performance of the system while the scaffolds and foams contribute to the mechanical stability of the ensemble.

In particular, the system of interest is the construct composed of a PEA scaffold, which can be prepared in varied porous morphologies as presented in [16,17], and the RAD16-I peptide. Herein we have targeted the characterization at a nanometric scale of the interaction between this SAP and the acrylic substrate used to produce the scaffolds and a 90/10 wt poly(ethyl acrylate-co-acrylic acid) copolymer as more hydrophilic material [19]. The interaction between PEA substrates and extracellular matrix proteins such as fibronectin [20,21] or laminin [22] has already been characterized: PEA has been found to favor the adhesion of these proteins and to facilitate the formation of a protein network that contributes to increase subsequent cell adhesion despite the hydrophobicity of the material. The fact that these mediating proteins are capable of spontaneously forming this network on its surface is an indicator of the potential biocompatibility of this material. Variations on the physico-chemical properties of the substrates such as wettability and chain mobility [23], or surface charge, for example, influence the resulting protein network [24]. Among others, atomic force microscopy (AFM) has shown to be a powerful tool to reveal such interactions. Given the similarities between those molecules and the SAPs, and since it has been previously used to characterize the SAPs morphology [5], AFM has also been the main technique of choice here.

Congo red is a specific dye for beta sheet structures, which is the secondary structure adopted by the peptide once its self-assembly occurs. It has been used as a rough assay to assess the presence and distribution of the peptide on the substrate surface. Next, AFM has been used to finely characterize 
the morphology and configuration adopted by the peptide on the substrate surface after applying two SAP deposition protocols, i.e., a set of samples have been observed directly after the adsorption of the SAP on the substrate, and another set after inducing the self-assembly of the SAP previously adsorbed. In that way not only the conformation and distribution but also the substrate influence on the self-assembly process have been studied. A basic vapor atmosphere has been used to promote the self assembly of the peptide, to avoid the dilution of the peptide solution and the presence of salts that would result from the assembly by addition of drops of phosphate-buffered saline (PBS) or culture medium. Finally, to elucidate the physics phenomenon promoting the different patterns of adsorption, contact angle measurements with different liquids and conditions have been undertaken.

\section{Materials and methods}

\section{1.- Materials}

\subsection{1.- Polymerization of acrylates}

Films of poly(ethyl acrylate) and a $90 \mathrm{wt} \%$ EA, $10 \mathrm{wt} \%$ AAc copolymer (P(EAcoAAc)) were obtained by ultraviolet polymerization. Briefly, ethyl acrylate (EA; 99\%, Sigma-Aldrich) and acrylic acid (AAc; 99\%, Sigma-Aldrich) were stirred at the given proportions with $1 \mathrm{wt} \%$ initiator (Benzoin, Scharlab) for $15 \mathrm{~min}$ at room temperature (RT). Then, the solutions were injected between two glass plates separated by a wire of $1.2 \mathrm{~mm}$ diameter, and placed in the oven for $8 \mathrm{~h}$ for the polymerization to occur. Subsequently, a thermal post-polymerization was carried out at $90^{\circ} \mathrm{C}$ for 24 hours. Afterwards, the obtained films were intensely dried under vacuum and temperature $\left(60^{\circ} \mathrm{C}\right)$ in order to remove any residual monomer trace remaining in the samples. 


\subsection{2.- Spin coating}

PEA films were dissolved in toluene (synthesis grade, Scharlab) to a final concentration of 2 wt $\%$ by stirring overnight. Analogously, P(EAcoAAc) films were dissolved in an 80/20 vol. toluene/dioxane (extra pure, Scharlab) mixture to a final concentration of $2 \mathrm{wt} \%$.

Then, ultra-thin polymer films were obtained on $12 \mathrm{~mm}$ diameter glass coverslips by spin casting $100 \mu \mathrm{L}$ of the polymer solutions at $3000 \mathrm{rpm}$ with an acceleration of $3000 \mathrm{rpm} / \mathrm{s}$ for $30 \mathrm{~s}$ in a spin coater (Brewer Science). Once prepared, samples were dried under vacuum and $60^{\circ} \mathrm{C}$ overnight to remove any trace of solvents.

\subsection{3.- Adsorption of the self assembling peptide}

The self-assembling peptide used was RAD16-I (Puramatrix, BD Biosciences). The SAP starting solution was sonicated for 30 minutes, and then diluted with ultradeionized water to a final concentration that varied depending on the experiment as follows. The stock solution was sonicated again prior to each use in order to disrupt the aggregates that could be formed during storage.

\section{a) Protocol 1:}

Spin coated PEA and P(EAcoAAc) samples were incubated with $100 \mu \mathrm{L}$ of SAP solution each for 1 hour at $37^{\circ} \mathrm{C}$, to allow the SAP to adsorb on the material surface. Different concentrations were used, ranging from $0.1 \%$ down to $0.05 \%$ and $0.025 \%$. To self-assemble the peptides, samples were transferred to a chamber with a $\mathrm{NaOH}(\mathrm{aq}) 12 \mathrm{M}$ basic ambient, and left in this atmosphere for 4 hours, analogously to the procedure followed in [25] with basic peptides that gel under acid vapor. Once the films were retrieved from the $\mathrm{NaOH}$ atmosphere, the excess of SAP solution was carefully removed with a paper, and then samples were gently rinsed with ultradeionized water. 


\section{b) Protocol 2:}

Spin coated PEA and P(EAcoAAc) samples were incubated with $100 \mu \mathrm{L}$ of SAP solution at a $0.025 \%$ concentration each, for 1 hour at $37^{\circ} \mathrm{C}$, to allow the SAP to adsorb on the material surface. Then, the excess of SAP solution was carefully removed with a paper, and samples were gently rinsed with ultradeionized water.

\section{2.- Methods}

\subsection{1.- Congo red staining}

In order to coarsely study the substrate-SAP interaction, surfaces with $0.25 \%$ SAP absorbed on them by following protocol 1 were stained before and after removing the SAP excess, by immersion in a $0.1 \% \mathrm{wt} / \mathrm{vol}$ congo red (Fisher) aqueous solution for $20 \mathrm{~min} .3$ replicates per substrate were stained. They were finally washed for $5 \mathrm{~min}$ with distilled water prior to their examination. Glass covers equally treated were used as controls.

\subsection{2.- Atomic force microscopy (AFM)}

After adsorption following any of the protocols, samples were dried for 30 min under an incandescent lamp, mounted and observed by atomic force microscopy (AFM). AFM images were obtained in a BRUKER Multimode 8 AFM device equipped with NanoScope IIIa controller (Digital Instruments-Veeco) operating in tapping mode in air. Silicon-cantilevers (Veeco, Manchester, UK) were used with force constant of $2.8 \mathrm{~N} / \mathrm{m}$ and resonance frequency of $75 \mathrm{kHz}$. The phase signal was set to zero at a frequency of 5-10\% lower than the resonance frequency of the tip, as suggested by the manufacturer. Drive amplitude was $600 \mathrm{mV}$ and the amplitude set point was $1.8 \mathrm{~V}$. The ratio $\mathrm{A}_{\mathrm{sp}} / \mathrm{A}_{0}$ was kept equal to 0.8 . 


\subsection{3.- Contact angle measurements}

In order to study the surface wettability of different materials (or a given material after different surface treatments), the contact angles of $3 \mu \mathrm{L}$ drops of water (extra pure, Scharlau), formamide (99.5\%, Aldrich) and diethylenglycol (99\%, Aldrich) on their surfaces were measured. A Dataphysics OCA instrument (DataPhysics Instruments GmbH, Filderstadt, Germany) was used for this purpose. Surfaces, either bare or with RAD16-I adsorbed on them, were measured in their dry state, or after swelling in a $100 \%$ relative humidity $(\mathrm{RH})$ atmosphere for 3 days, to elucidate the influence of maintaining the samples in $\mathrm{RH}$ (as occurs during the preparation of AFM samples with RAD16-I adsorbed). Besides, as support in understanding the immediate interaction of the SAP with the polymers, the contact angle of $3 \mu \mathrm{L}$ drops of the SAP solution at $0.1 \%$ and $0.025 \%$ concentrations was also determined. Measurements were carried out at least 10 times for each liquid, material composition and treatment.

\section{Results}

\subsection{Congo red staining}

Congo red staining was first applied onto bare spin coated PEA and P(EAcoAAc) and glass covers to be used as controls, to discard their coloration. As can be observed in Fig. 1 A, D and G, none of the samples acquired any redish tonality.

When surfaces where RAD16-I had been previously adsorbed, self-assembled and its excess carefully removed, were stained with this dye, a very light red shade was left for all the materials under study (Fig. 1 B, E and H). This coloring clearly reveals the presence of the peptide on the surfaces. Interestingly, the glass cover seemed to acquire a stronger coloration. No differences were detected, though, between the PEA and P(EAcoAAc) polymers. 
However, congo red staining revealed that when RAD16-I is adsorbed, self-assembled but its excess is not removed from the surface, a thin three-dimensional gel is formed on all surfaces. This layer is weakly attached, and during the washes following the staining process some parts might detach, as shown in Fig. $1 \mathrm{~F}$ and I. No differences were again found here between the two polymers under study despite their different composition and hydrophilicity degree. Once more, glass covers seemed to give rise to a more uniform and stable layer.

\subsection{Atomic force imaging}

a) Bare substrates

By means of AFM images a more exhaustive characterization of the SAP-substrate interaction was performed. As a first step, bare materials were scanned to characterize their surfaces, to subsequently identify and distinguish them from the conformation acquired by the adsorbed peptide. As can be observed in Fig. 2, glass covers present a characteristic surface roughness that is not detected in the PEA and P(EAcoAAc) spin coated films. This is an indicator of the success in obtaining a uniform and continuous polymeric layer on the cover.

\section{b) Samples prepared following protocol 1}

When RAD16-I was adsorbed on PEA and P(EAcoAAc) surfaces at a concentration of $0.1 \%$, a very compact and dense block of peptide adsorbed on the materials surface was observed (Fig. 3). However, given the high density of nanofibrils even at such low concentration (RAD16-I is usually used at concentrations of $0.15-0.25 \%$ ), it was impossible to distinguish and establish any difference between the conformation or distribution of the peptide because of the different nature of the tested materials. It was decided thus to reduce the concentration of the peptide solution from this point on. 
When the surfaces were coated with a half diluted solution (i.e., 0.05\%) differences between them started to be unveiled. As shown in Fig. 4, a uniform coating, with a homogeneous fiber distribution was formed on covers. In the case of PEA, randomly distributed gaps and empty spaces are left as the SAP network forms. As for the $\mathrm{P}(\mathrm{EAcoAAc})$, a continuous coating of the surface is also observed; however, in the $3 \mu \mathrm{m}$ window the resulting structure seems to not be flat, but to present some valleys. Nonetheless, these valleys, unlike the PEA gaps, are uniformly coated with peptide nanofibres as the rest of the film surface. When $500 \mathrm{~nm}$ windows are compared, great differences can be detected between the distribution of the self-assembled nanofibres on PEA and P(EAcoAAc) substrates. Covers and $\mathrm{P}(\mathrm{EAco} A \mathrm{Ac})$ exhibit a more similar distribution, though both with a very dense concentration of nanofibrils that hinders getting more information about the substrate influence; for this reason, the concentration of the SAP solution was halved once more, to $0.025 \%$.

As can be observed in Fig. 5, when the RAD16-I is diluted up to 0.025\%, the differences between these materials get clearer. In the $3 \mu \mathrm{m}$ images one can see that the SAP does not reach to form uniform layers on PEA, unlike on the cover and $\mathrm{P}(\mathrm{EAcoAAc})$, where a uniform layer of peptide is yet obtained. At greater magnifications, differences in the resulting net can be more clearly seen: on the covers, the RAD16-I self-assembles into straight nanofibres that form a very dense net over the entire surface, whereas the peptide self-assembles into a clearer network on P(EAcoAAc), forming circular units consisting in straight nanofibres. A less dense network is obtained on PEA, with a more disorganized structure without any pattern. The $1 \mu \mathrm{m}$ height images were characterized in terms of the typical texture parameters by using the Gwyddion software for SPM (scanning probe microscopy) data visualization and analysis (Department of Nanometrology, Czech Metrology Institute), and are shown in Table 1. These results illustrate better the greater density (translated into 
roughness) of the coating formed on covers, and the uniformity of that on the copolymer as compared with the homopolymer, which displays thus an intermediate root mean square roughness.

\section{c) Samples prepared following protocol 2}

Since the most revealing results were obtained for the lowest SAP concentration, protocol 2 was followed with the $0.025 \%$ SAP solution. The differences among the substrates coated according to protocol 1, are seen as well under protocol 2, despite their different preparation. On PEA substrates, a non-uniform coating, leaving spaces and empty gaps was obtained, while on P(EAcoAAc) ones a uniform layer of nanofibres is displayed throughout the surface. Altogether, the copolymer seems to be a more sensitive substrate to the preparation protocol since greater differences between Fig. 5 and 6 are detected for this material. Again for the covers the most uniform and dense network of fibres was obtained. The textural parameters of these images are also displayed in Table 1, and show an analogous trend to that of samples treated following protocol 1.

\subsection{Contact angle measurements}

The differences found on the AFM images were thought to be attributable to differences in the wettability of the materials' surfaces. For this reason, a thorough study of the contact angles was undertaken (Fig. 7). The highest water contact angle was obtained for PEA $\left(90^{\circ}\right)$; the addition of acrylic acid units in the $90 / 10$ copolymer $\mathrm{P}(\mathrm{EAcoAAc})$ induced its decrease to $75^{\circ}$, whereas that of the bare cover was $46^{\circ}$. For diethylenglycol, a dramatic reduction on the mean value was observed: $63^{\circ}, 56^{\circ}$ and $27^{\circ}$, respectively. A more moderate drop was observed for formamide, which has a polarity between those of water and diethylenglycol, PEA reaching a value of $76^{\circ}, 67^{\circ}$ for the copolymer and $42^{\circ}$ for the covers. 
The effect of the SAP on each surface on the wettability was evaluated by measuring the contact angles again on the substrates after formerly adsorbing RAD16-I following protocol 1. For PEA and covers, a clear trend was observed: the adsorption of RAD16-I on the surface prior to the measurement slightly reduced the obtained contact angles, to a different extent depending on the liquid. Differently, for $\mathrm{P}(\mathrm{EAcoAAc})$ the lower water contact angle increased (from $75^{\circ}$ up to $89^{\circ}$ ) after the peptide adsorption, whereas for the other, less polar [26,27], liquids a slight decrease was again observed.

Besides, the RAD16-I contact angle was measured at different concentrations, on dry substrates or on substrates previously swollen to equilibrium in an atmosphere of $100 \%$ relative humidity. Neither for PEA nor for P(EAcoAAc), significant differences were found when changing the SAP concentration (Fig. 8), the contact angle being around $94^{\circ}$. In both cases, the peptide contact angle is higher than that of water, no matter the previous conditioning of the substrates. Differently, for the glass covers the peptide contact angle was slightly lower than that of pure water. When swollen, both PEA and P(EAcoAAc) showed a reduction of the contact angle for the three liquids used. Such behavior was surprisingly the opposite for the 'swollen' covers, on which increases of the contact angles were found.

\section{Discussion}

\section{On the acrylates interaction with RADI6-I}

Congo red dye is commonly used for the characterization of beta-sheet structures in general (like diagnosis of diseases related with this type of structures), but also for the characterization of selfassembling peptides like Q11 [28] or RAD16-I [29], which adopt this specific structure as well. The initial characterization of the peptide interaction with the polymers under study was for this reason 
performed with this dye. This technique allowed verifying that the process followed successfully permitted the adsorption of the peptide on both polymers surfaces and covers (Fig. 1).

The problematic associated to using this technique, is that after the excess of the SAP gel is removed samples only acquire a very clear red shade. Covers seem to exhibit the strongest coloration, but anyway too clear to allow establishing supported differences between the spin coated acrylates. The removal of excess of peptide solution ensues though another setback. The SAPs form a dense network that is only attached to the surface of the polymer film or cover by the interaction of the first layer of the peptide in contact with the substrate; therefore, a great mass is attached to the surface by electrostatic interactions of just a few molecules. As a consequence, portions of the gel can be easily removed and washed out during the staining process (images $\mathrm{C}$ and $\mathrm{F}$ in Fig. 1). Taken all this together, the congo red staining was found to be a coarse technique for the level of detail and accuracy required to settle differences between acrylates of different hydrophilicity in their interactions with the aqueous SAP. AFM allowed to study such correlation with greater detail; this technique has indeed already proved to be very useful in the determination of proteins conformation and interaction with polymeric substrates [30,31].

When the concentration of the RAD16-I used in protocol 1 was low enough, differences in the conformation adopted by the peptide onto the different substrates were uncovered. A clear relationship was found between the better spreading of the peptide on the material and the percentage of electronegative atoms present in the substrate. Since the isoelectric point of RAD16-I is 7.2 [32], the net charge of the aqueous peptide during the adsorption stage is positive in both protocols. For PEA, in which functional groups the differences in electronegativity are less pronounced than in its copolymer with acrylic acid, the wettability was poorer and as a result the SAP assembled and percolated leaving empty spaces and bare areas. In P(EAcoAAc), the 
incorporation of $10 \%$ of monomeric units containing the carboxyl group favored the formation of a more uniform and regular coating on the surface. However, circular structures leaving empty spaces were yet observed. In glass covers, the presence of negatively charged oxygen atoms promote a better interaction during the adsorption stage with the amine groups in the aqueous peptide solution that completely wets the surface; as a result, a very dense layer of peptide forms and bare areas are not observed any more. This rationale is in good agreement with the water contact angle results (Fig. 7 and 8): the highest value was obtained for PEA, a bit lower for P(EAcoAAc), and the lowest for covers, which precisely are the most uniformly coated by the SAP.

The influence of the electronegativity and wettability of the substrate was similarly revealed through protocol 2, which differed from protocol 1 in that assembly was not induced after the adsorption stage. More specifically, the resulting peptide coating obtained herein seemed more sensitive to these factors since greater differences were detected between PEA and its copolymer with acrylic acid (Fig. 6): a denser and more uniform layer of fibers was formed on the copolymer, while for the analogous samples prepared following protocol 1 (Fig. 5) some gaps and empty spaces were still observed despite its wettability. Another interesting finding from this experiment was that despite not adding any salt or increasing the $\mathrm{pH}$ of the ambient, the peptide was able to interact to generate fibrils and a percolated network, which suggests that the chemistry and physical state (vitreous for the covers and rubber for acrylates) of three substrates are able to trigger the selfassembling of the peptide by themselves. Indeed, PEA based copolymers containing hydroxyl groups have already proven to trigger the formation of fibronectin networks on their surfaces, provided that the fraction of the latter be lower than 0.7 [17]. Other studies have also demonstrated that the presence of carboxyl groups enhanced the protein surface interaction [33], which had a straightforward impact on the biological outcome of the substrate [34]. 
The adsorption of the peptide was found to induce a reduction in the contact angle in general, no matter the polarity of the solvent used and the polymeric substrate, except the copolymer, for which the wettability with water, the most polar solvent used, decreased after the peptide coating. Therefore, the presence of the peptide alters the wettability of the polymeric substrate, which will eventually have an effect on the way and extent proteins from the medium will adsorb, and the way cells adhere and interact with it [35].

It has to be mentioned that a certain discrepancy was detected between the contact angle values here presented and the values found elsewhere [36]. These differences are to be attributed to the preparation of the substrates, being crosslinked films in the previously published results, and ultrathin spin coated films of non-crosslinked polymers in the present work. Herein, polymeric chains are stretched because of the centripetal force applied to obtain them. Having the chains in such a particular disposition might induce certain interactions among the chains' dipoles that when they are in a random coil configuration do not take place. These changes of conformation and availability of hydrophilic domains translate into different wettability, contact angle and surface free energy values as presented.

As already stated, PEA reached the greatest water contact angle as shown in Fig 7., indicating a poorer wettability than the other substrates. Nonetheless, when the RAD16-I contact angles were measured at different concentrations, both PEA and P(EAcoAAc) were found to shed very similar values, whereas such solution seemed to have a much better interaction with bare covers because they exhibited a much lower water contact angle. This similarity between PEA and P(EAcoAAc) in terms of their RAD16-I contact angles is somehow in conflict with their AFM images, where P(EAcoAAc) was notably better wet than PEA (Fig. 2 to Fig. 5). To elucidate the cause of such despair results, the RAD16-I (at two concentrations) and water contact angle were measured again 
but this time with samples previously swollen under a controlled relative humidity. This experiment allowed taking into account the fact that the adsorption and self-assembly of the peptide prior to the AFM observation is performed under wet conditions and measuring dry substrates was not realistic. PEA and P(EAcoAAc) showed a decrease of the water contact angle because of their pre-swelling and now the $\mathrm{P}(\mathrm{EAcoAAc})$ copolymer did yield the lowest values, in agreement with its better interaction seen on AFM images. Surprisingly, for the covers, the contact angles of the swollen samples were higher; a plausible cause could be found in the interaction of water molecules (from the wet atmosphere), with the electronegative groups of the cover surface which therefore are no longer available to interact with water or peptide solutions. Nonetheless, the reached values are much lower than those measured on acrylates, which is in good agreement with the AFM images, which show a more uniform coating than the latter.

In order to get a deeper insight, after the contact angle measurements, the surface tension of the materials was calculated using the Young equation in combination with the Owens Wendel Raelble and Kaelble equation, OWRK [37], Table 2.

The introduction of carboxyl groups in $\mathrm{P}(\mathrm{EAcoAAc})$ translates into a greater polar component than that of bare PEA, and the most polar substrate was the glass cover, as expected. For PEA, a lower surface free energy and greater polar component than those previously published values $[38,39]$ were obtained here; as explained, these discrepancies can be a side effect of the production procedure.

For PEA and glass covers the previous adsorption of RAD16-I provoked an increase of the polar component that increased the global surface free energy. The opposite occurred with P(EAcoAAc), for which the adsorption of the peptide decreases the wettability by polar substances of the exposed area, while the hydrophilicity of the bulk is kept constant as dictated by its chemistry. 


\section{Conclusions}

The substrate/RAD16-I interaction was found to be strongly dependent on the substrate hydrophilicity and surface wettability. The PEA hydrophobic nature provided a poorer interaction with the peptide than its copolymer with acrylic acid, P(EAcoAAc), whose $10 \%$ AAc hydrophilic groups improved significantly the interplay to allow a percolated nanofibrils network that coated entirely the surface and decreased its surface tension. Interestingly, the chemistry of both substrates proved to be capable to trigger the self-assembly of the peptide by themselves without the need of a $\mathrm{pH}$ increase or the addition of salts (PBS or culture medium, for instance). This finding opens up new possibilities for the acrylates/RAD16-I combination in tissue engineering strategies.

\section{Acknowledgements}

The authors acknowledge funding through the European Commission FP7 project RECATABI (NMP3-SL-2009-229239), and from the Spanish Ministerio de Ciencia e Innovación through projects MAT2011-28791-C03-02 and -03. This work was also supported by the Spanish Ministerio de Educación through M. Arnal-Pastor FPU 2009-1870 grant. The authors acknowledge the assistance and advice of Electron Microscopy Service of the UPV. 


\section{Figures}
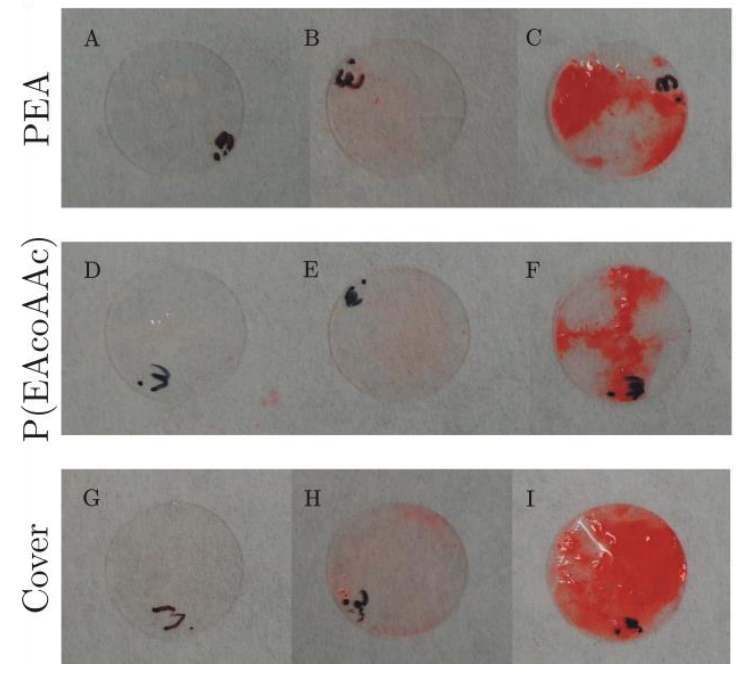

Fig 1: PEA and P(EAcoAAc) spin coated samples, and glass covers (A, D, G), with RAD16-I adsorbed on the surface stained with congo red after (B, E, H) and before (C, F, I) removing the SAP excess.

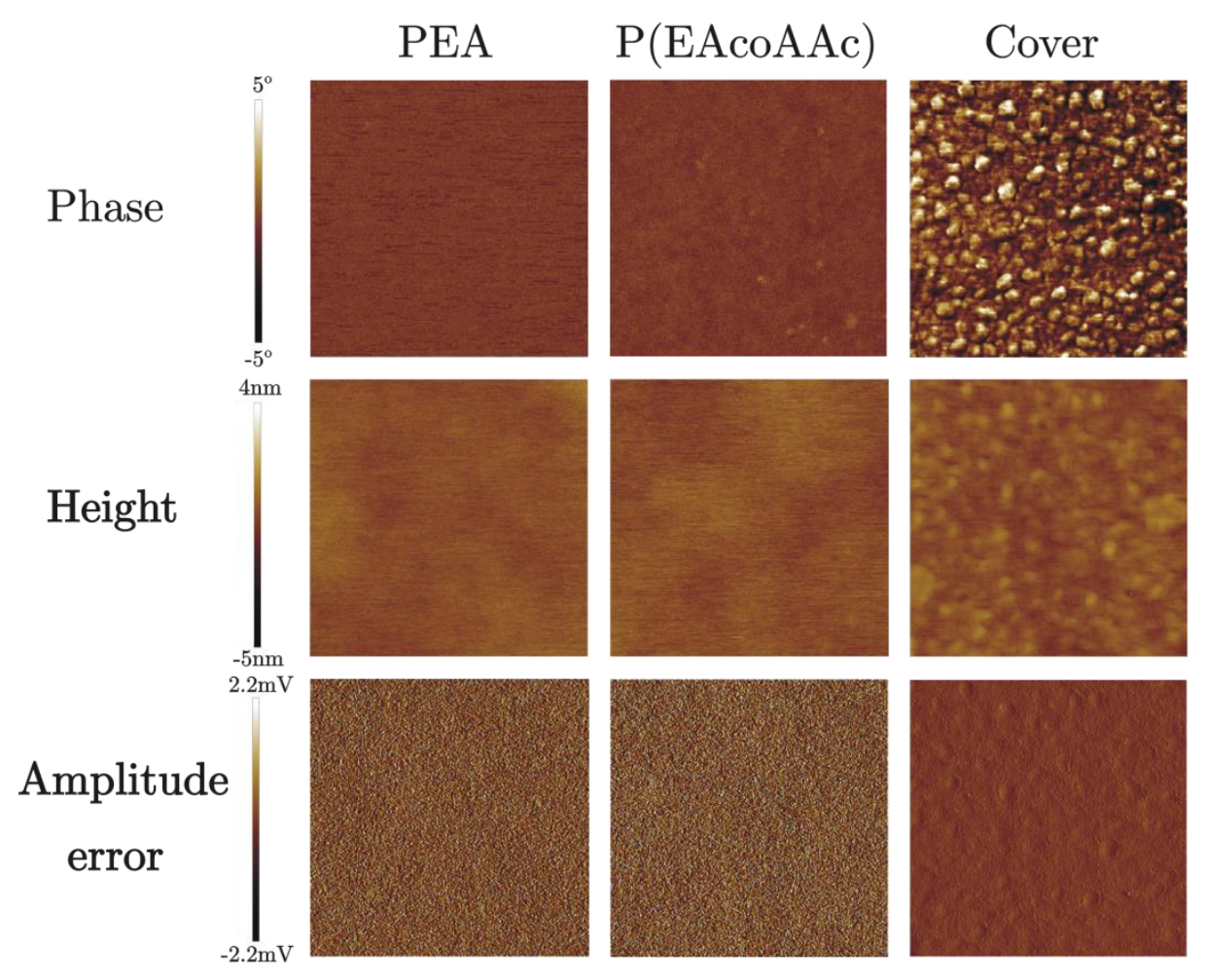

Fig. 2: AFM images of spin coated PEA and P(EAcoAAc) films and bare glass covers. Phase (first row), height (second row) and amplitude error (third row) AFM magnitudes, shown as $500 \mathrm{~nm}$ side windows. 


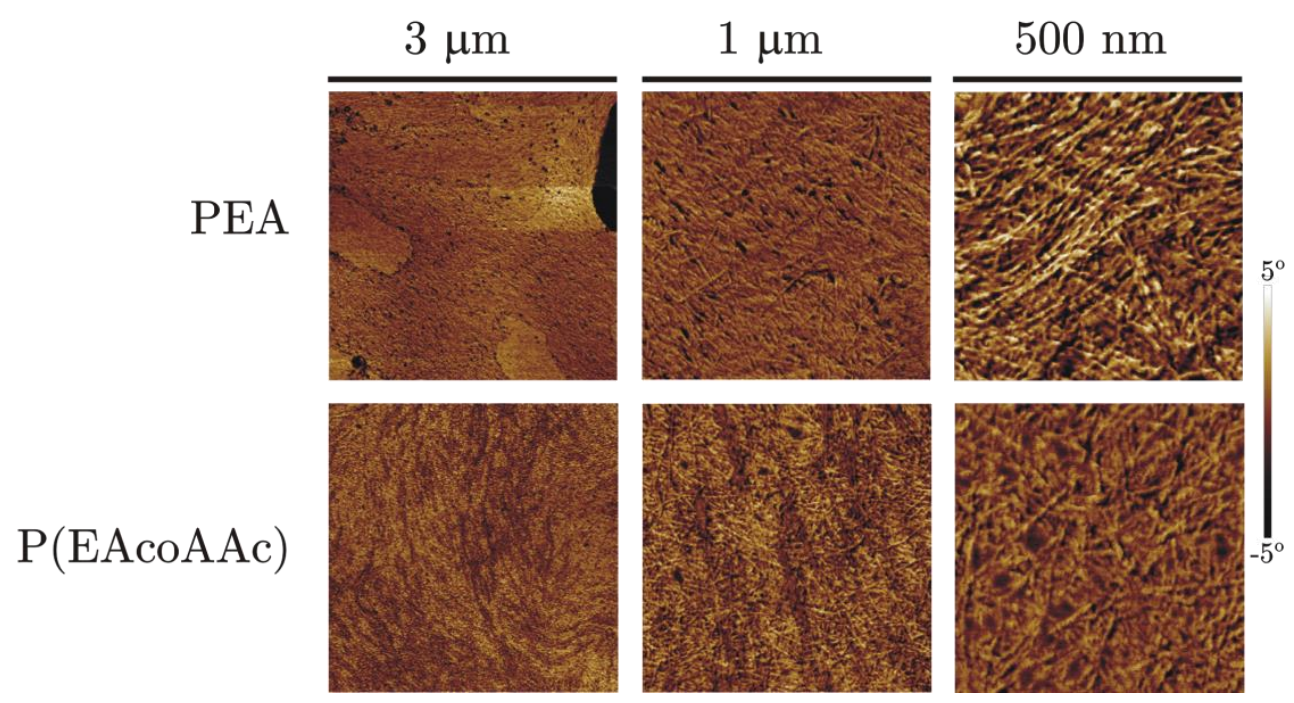

Fig. 3: Conformation and distribution of the SAP adsorbed at a $0.1 \%$ concentration on the surface of PEA (first row) and $\mathrm{P}(\mathrm{EAcoAAc}$ ) (second row) and gelled in a basic ambient (protocol 1), as shown by the phase AFM magnitude.

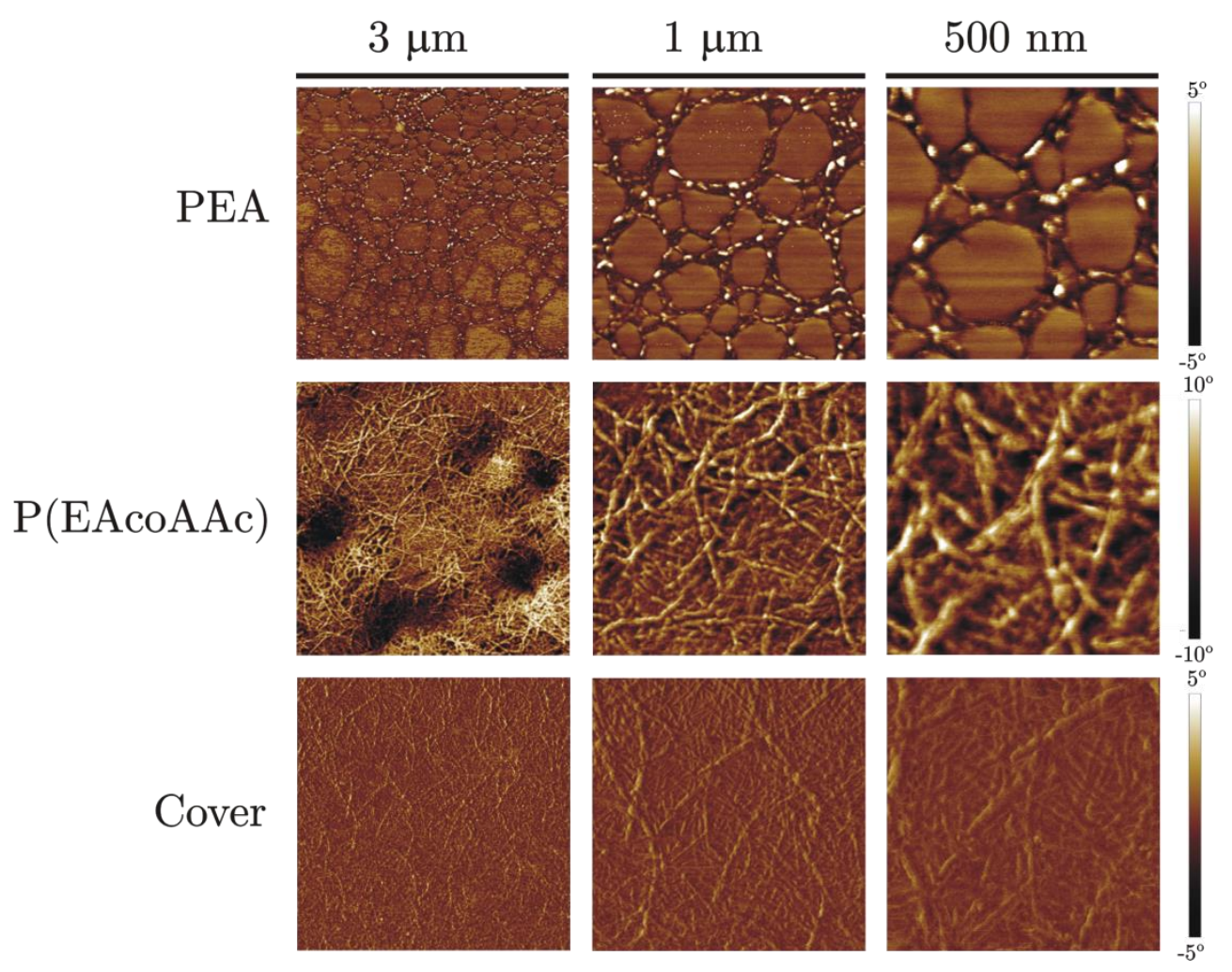

Fig. 4: Conformation and distribution of the SAP adsorbed at a $0.05 \%$ concentration on the surface of PEA (first row),

$\mathrm{P}(\mathrm{EAcoAAc}$ ) (second row) and covers (third row) and gelled in a basic ambient (protocol 1), for the phase AFM magnitude in $3 \mu \mathrm{m}$ windows (first column), $1 \mu \mathrm{m}$ windows (second column) and $500 \mathrm{~nm}$ windows (third column). 


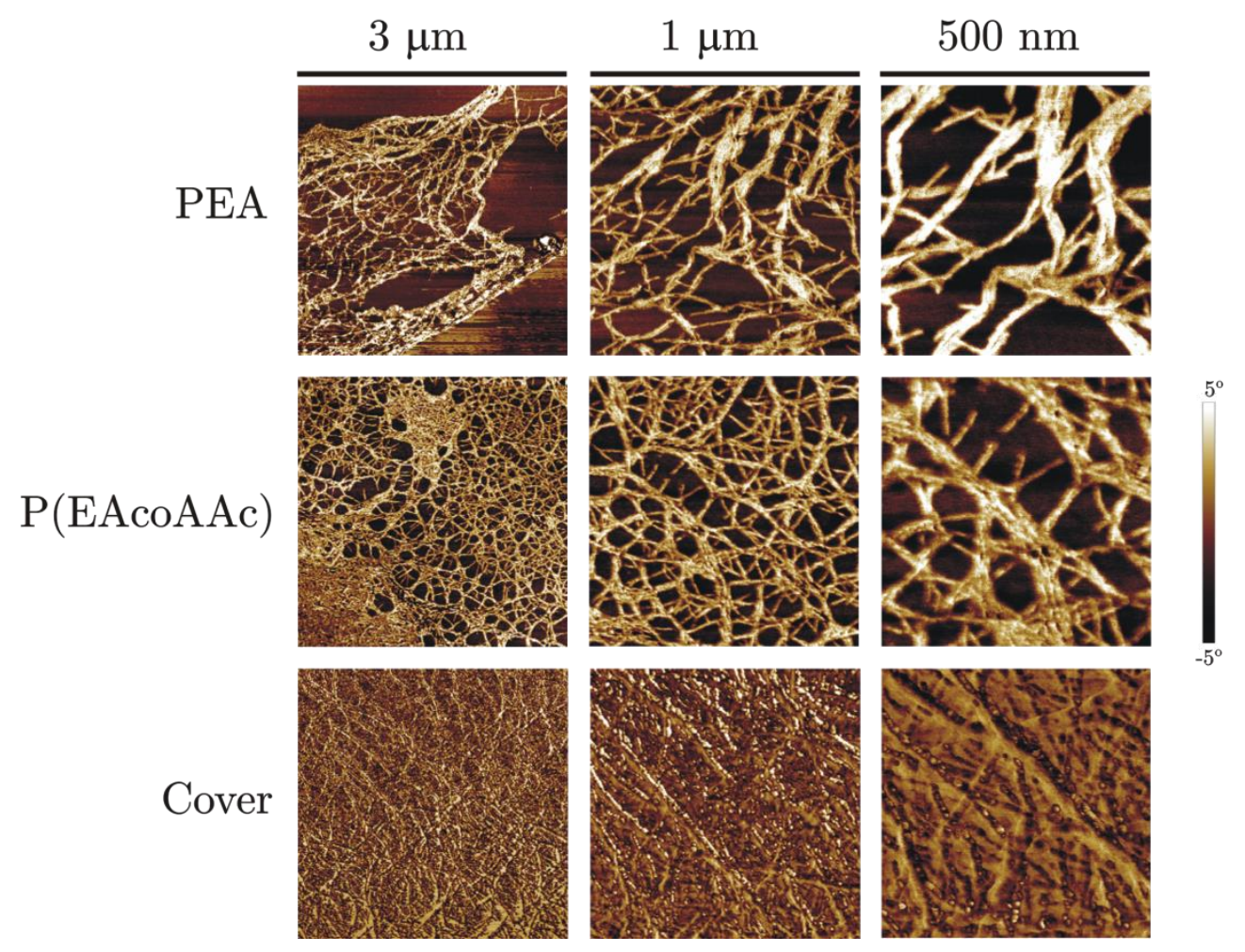

Fig. 5: Conformation and distribution of the SAP adsorbed at a $0.025 \%$ concentration on the surface of PEA (first row),

$\mathrm{P}(\mathrm{EAcoAAc}$ ) (second row) and covers (third row) and gelled in a basic ambient (protocol 1), for the phase AFM magnitude in $3 \mu \mathrm{m}$ windows (first column), $1 \mu \mathrm{m}$ window (second column) and $500 \mathrm{~nm}$ window (third column). 


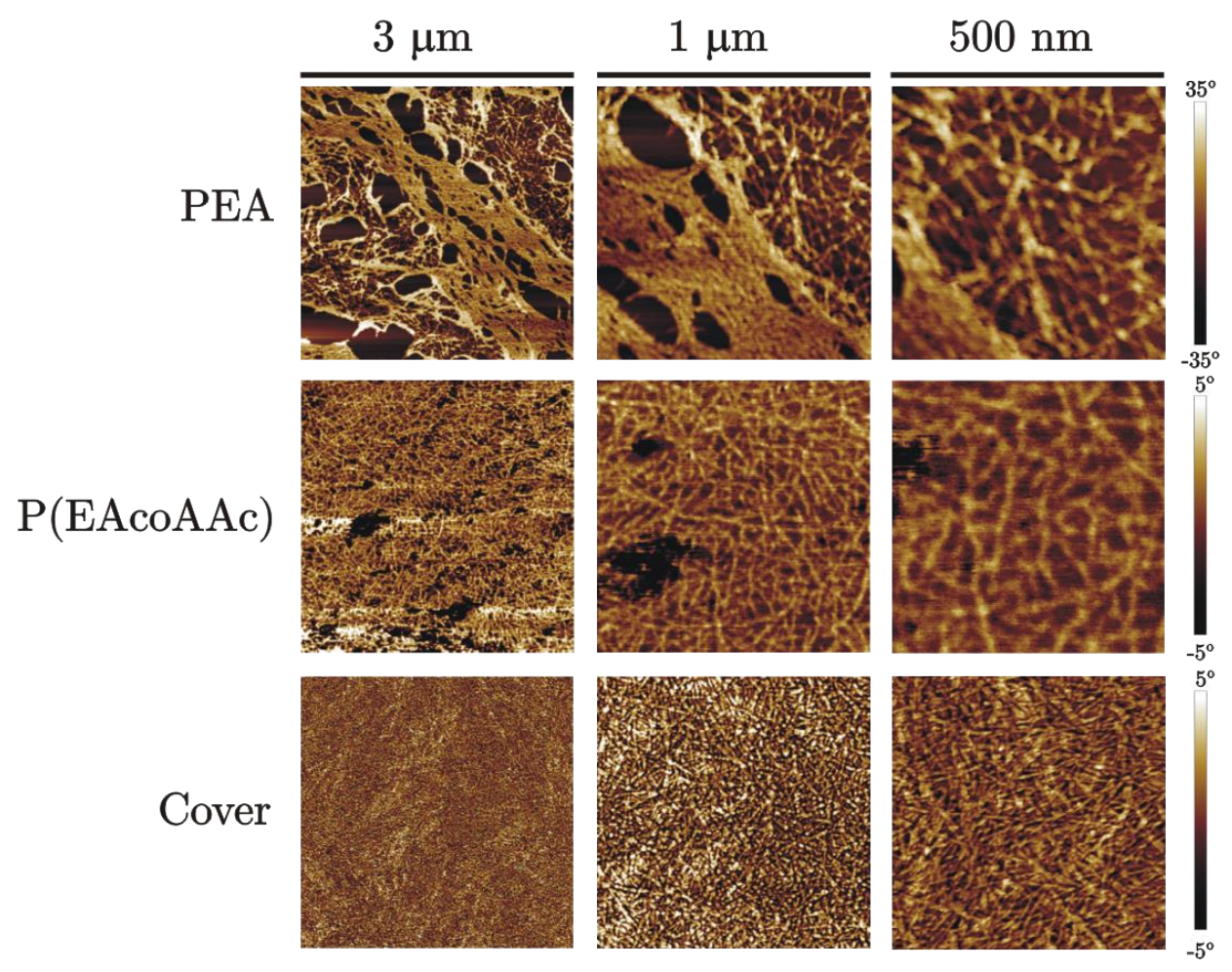

Fig. 6: Conformation and distribution of the SAP adsorbed at a $0.025 \%$ concentration (protocol 2) on the surface of PEA (first row), P(EAcoAAc) (second row) and covers (third row), for the phase AFM magnitude in $3 \mu \mathrm{m}$ windows (first column), $1 \mu \mathrm{m}$ window (second column) and $500 \mathrm{~nm}$ window (third column).

$\mathrm{H}_{2} \mathrm{O}$ Bare $\square \mathrm{H}_{2} \mathrm{O}$ SAP Diethylenglycol Bare Diethylenglycol SAP Formamide Bare $\mathbb{N}$ Formamide SA P

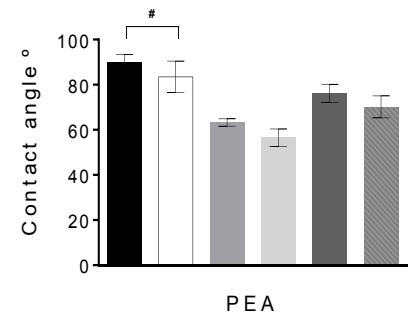

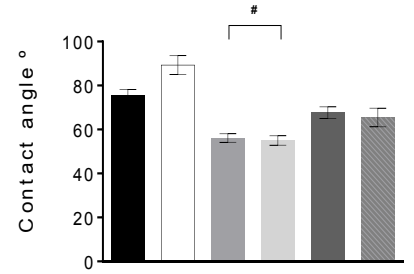

$P(E A \operatorname{coA} A c)$

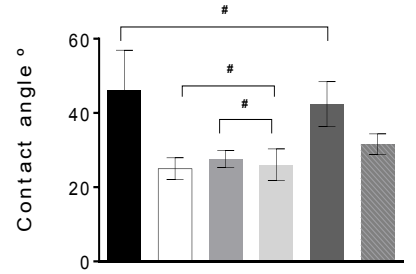

Cover

Fig. 7: Water, diethylenglycol and formamide contact angles on bare and SAP coated PEA, P(EAcoAAc) and covers. (\#) Differences are not statistically significant. 


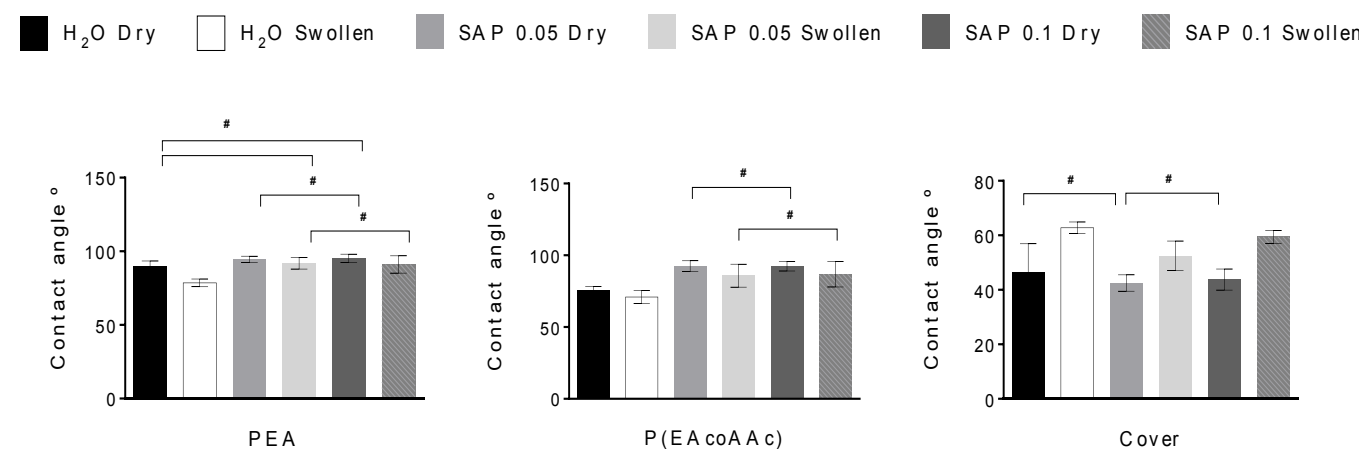

Fig. 8: Water and RAD16-I (at 0.05 and $0.1 \%$ ) contact angles on bare PEA, P(EAcoAAc) and covers, either dry or swollen in a $100 \%$ relative humidity atmosphere. \# Differences are not statistically significant. 
Tables

\begin{tabular}{ccccc}
\hline material & RMS & $\mathbf{R}_{\mathbf{T}}$ & $\mathbf{R}_{\mathbf{V}}$ & $\mathbf{R}_{\mathbf{P}}$ \\
\hline PEA (protocol 1) & 0.70 & 14.38 & 1.82 & 2.94 \\
P(EAcoAAC (protocol 1) & 0.23 & 6.00 & 0.71 & 0.61 \\
cover (protocol 1) & 1.17 & 20.95 & 2.91 & 3.25 \\
PEA (protocol 2) & 0.54 & 10.36 & 1.69 & 1.49 \\
P(EAcoAAC (protocol 2) & 0.15 & 4.76 & 0.35 & 0.33 \\
cover (protocol 2) & 1.24 & 14.03 & 2.72 & 2.80 \\
\hline
\end{tabular}

Table 1: Root mean square roughness (RMS), maximum height of the profile $\left(\mathrm{R}_{\mathrm{T}}\right)$, maximum roughness valley depth $\left(\mathrm{R}_{\mathrm{V}}\right)$ and maximum roughness peak height $\left(\mathrm{R}_{\mathrm{P}}\right)$, in nm, obtained from AFM height images.

\begin{tabular}{ccccc}
\hline material & $\boldsymbol{\sigma}^{\mathbf{d}}(\mathbf{m} \mathbf{N} / \mathbf{m})$ & $\boldsymbol{\sigma}^{\mathbf{p}}(\mathbf{m N} / \mathbf{m})$ & $\boldsymbol{\sigma}(\mathbf{m N} / \mathbf{m})$ & polarity $\left(\boldsymbol{\sigma}^{\mathbf{p}} / \boldsymbol{\sigma}\right)$ \\
\hline cover (bare) & 5.28 & 4.44 & 9.72 & 0.46 \\
cover / SAP & 5.38 & 5.14 & 10.52 & 0.49 \\
PEA (bare) & 5.48 & 1.17 & 6.65 & 0.18 \\
PEA / SAP & 5.53 & 1.68 & 7.21 & 0.23 \\
P(EAcoAAc) (bare) & 5.68 & 1.97 & 7.65 & 0.26 \\
P(EAcoAAc) / SAP & 6.07 & 1.13 & 7.2 & 0.16 \\
\hline
\end{tabular}

Table 2: Polar $\left(\boldsymbol{\sigma}^{\mathbf{p}}\right)$ and dispersive $\left(\boldsymbol{\sigma}^{\mathbf{d}}\right)$ components of the surface tension, surface free energy $(\boldsymbol{\sigma})$ and polarity $\left(\boldsymbol{\sigma}^{\mathbf{p}} / \boldsymbol{\sigma}\right)$ of the substrates, bare or coated with RAD16-I. 


\section{References}

1. Davis ME, Motion JP, Narmoneva DA, Takahashi T, Hakuno D, Kamm RD, Zhang S, Lee RT. Injectable self-assembling peptide nanofibers create intramyocardial microenvironments for endothelial cells. Circulation 2005; 111(4): 442-450.

2. Zhang S, Lockshin C, Cook R, Rich A. Unusually stable beta-sheet formation in an ionic selfcomplementary oligopeptide. Biopolymers 1994; 34: 663-672.

3. Zhang S, Altman M. Peptide self-assembly in functional polymer science and engineering. Reactive \& Functional Polymers 1999; 41: 91-102.

4. Zhang S, Gelain F, Zhao X. Designer self-assembling peptide nanofiber scaffolds for 3D tissue cell cultures. Semin Cancer Biol. 2005; 15(5): 413-20.

5. Zhang S, Zhao X, Spirio L. PuraMatrix: Self-assembling peptide nanofiber scaffolds. In: Ma PX, Elisseeff J, eds. Scaffolding in tissue Engineering. Boca Raton, FL: 2005 CRC Press. pp. 217238.

6. Sieminski AL, Semino CE, Gong H, Kamm RD. Primary sequence of ionic self-assembling peptide gels affects endothelial cell adhesion and capillary morphogenesis. J Biomed Mater Res A. 2008; 87(2):494-504.

7. Quintana L, Fernández Muiños T, Genove E, Del Mar Olmos M, Borrós S, Semino CE. Early tissue patterning recreated by mouse embryonic fibroblasts in a three-dimensional environment. Tissue Eng Part A. 2009; 15(1): 45-54.

8. Garreta E, Genové E, Borrós S, Semino CE. Osteogenic differentiation of mouse embryonic stem cells and mouse embryonic fibroblasts in a three-dimensional self-assembling peptide scaffold. Tissue Eng. 2006; 12(8): 2215-2227.

9. Semino, C. E., Merok, J. R., Crane, G. G., Panagiotakos, G. \& Zhang, S. Functional differentiation of hepatocyte-like spheroid structures from putative liver progenitor cells in threedimensional peptide scaffolds. Differentiation 2003; 71: 262-270. 
10. Thonhoff JR, Lou DI, Jordan PM, Zhao X, Wu P. Compatibility of human fetal neural stem cells with hydrogel biomaterials in vitro. Brain Res. 2008; 1187: 42-51.

11. Tokunaga M, Liu ML, Nagai T, Iwanaga K, Matsuura K, Takahashi T, Kanda M, Kondo N, Wang P, Naito AT, Komuro I. Implantation of cardiac progenitor cells using self-assembling peptide improves cardiac function after myocardial infarction. J Mol Cell Cardiol. 2010; 49(6): 972983.

12. Takei J. 3-Dimensional Cell Culture Scaffold For Everyone: Drug Screening.Tissue Engineering and Cancer Biology. AATEX 2006; 11(3): 170-176.

13. McGrath AM, Novikova LN, Novikov LN, Wiberg M. BD ${ }^{\mathrm{TM}}$ PuraMatrix ${ }^{\mathrm{TM}}$ peptide hydrogel seeded with Schwann cells for peripheral nerve regeneration. Brain Res Bull 2010; 83(5): 207-213. 14. Wang W, Itoh S, Matsuda A, Aizawa T, Demura M, Ichinose S, Shinomiya K, Tanaka J. Enhanced nerve regeneration through a bilayered chitosan tube: The effect ofintroduction of glycine spacer into the CYIGSR sequence. J Biomed Mater Res Part A 2008: 85; 919-928.

15. Sargeant TD, Guler MO, Oppenheimer SM, Mata A, Satcher RL, Dunand DC, Stupp SI. Hybrid bone implants: self-assembly of peptide amphiphile nanofibers within porous titanium. Biomaterials 2008; 29(2): 161-171.

16. Vallés-Lluch A, Arnal-Pastor M, Martínez-Ramos C, Vilariño-Feltrer G, Vikingsson L, Castells-Sala C, Semino CE, Monleón Pradas M. Combining self-assembling peptide gels with three-dimensional elastomer scaffolds. Acta Biomaterialia 2013; 9(12): 9451-9460.

17. Valles-Lluch A, Arnal-Pastor M, Martinez-Ramos C, Vilarino-Feltrer G, Vikingsson L, Monleon Pradas M. Grid polymeric scaffolds with polypeptide gel filling as patches for infarcted tissue regeneration. Conf Proc IEEE Eng Med Biol Soc 2013; 2013: 6961-6964.

18. Soler-Botija C, Bagó JR, Llucià-Valldeperas A, Vallés-Lluch A, Castells-Sala C, MartínezRamos C, Fernández-Muiños T, Chachques JC, Monleón Pradas M, Semino CE, Bayes-Genis A. Engineered 3D bioimplants using elastomeric scaffold, self-assembling peptide hydrogel, and 
adipose tissue-derived progenitor cells for cardiac regeneration. Am J Transl Res 2014; 6(3): 291301.

19. Martínez-Ramos M, Arnal-Pastor M, Vallés-Lluch A, Monleón Pradas M. Peptide gel in a scaffold as a composite matrix for endothelial cells. J Biomed Mater Res Part A 2015; 103A: 32933302.

20. Rico P, Rodríguez Hernández JC, Moratal D, Altankov G, Monleón Pradas M, SalmerónSánchez M. Substrate-induced assembly of fibronectin into networks: influence of surface chemistry and effect on osteoblast adhesion. Tissue Eng Part A 2009; 15(11): 3271-3281.

21. Gugutkov D, Altankov G, Rodríguez Hernández JC, Monleón Pradas M, Salmerón Sánchez M. Fibronectin activity on substrates with controlled -OH density. J Biomed Mater Res A 2010; 92(1): 322-331.

22. Rodríguez Hernández JC, Salmerón Sánchez M, Soria JM, Gómez Ribelles JL, Monleón Pradas M. Substrate chemistry-dependent conformations of single laminin molecules on polymer surfaces are revealed by the phase signal of atomic force microscopy. Biophys J 2007; 93(1): 202207.

23. Cantini M, Rico P, Moratal D, Salmerón-Sánchez M. Controlled wettability, same chemistry: biological activity of plasma-polymerized coatings. Soft Matter 2012; 8: 5575-5584.

24. Anselme K, Ponche A, Bigerelle M. Relative influence of surface topography and surface chemistry on cell response to bone implant materials. Part 2: biological aspects. Proceedings of the Institution of Mechanical Engineers, Part H: Journal of Engineering in Medicine 2010; 224: 14871507.

25. Hartgerink JD, Beniash E, Stupp SI. Peptide-amphiphile nanofibers: a versatile scaffold for the preparation of self-assembling materials. Proc Natl Acad Sci USA 2002; 99(8): 5133-5138.

26. Busscher HJ, Vanpelt AWJ, Deboer P, Dejong HP, Arends J. The effect of surface roughening of polymers on measured contact angles of liquids. Colloids Surf 1984; 9: 319-331. 
27. Birdi KS. Surface tension of polymers. In: Yildrim Erbil H, ed. Handbook of surface and colloid chemistry. CRC Press, Boca Raton, 1997. p. 292.

28. Collier JH, MessersmithPB.Enzymatic Modification of Self-Assembled Peptide Structures with Tissue Transglutaminase.Bioconjug Chem. 2003; 14(4): 748-755.

29. Kakiuchi Y, Hirohashi N, Murakami-Murofushi K. The macroscopic structure of RADA16 peptide hydrogel stimulates monocyte/macrophage differentiation in HL60 cells via cholesterol synthesis. BiochemBiophys Res Commun. 2013; 433(3): 298-304.

30. Pérez-Garnes M, González-García C, Moratal D, Rico P, Salmerón-Sánchez M. Fibronectin distribution on demixednanoscale topographies. Int J Artif Organs. 2011; 34(1): 54-63.

31. Salmerón-Sánchez M, Rico P, Moratal D, Lee TT, Schwarzbauer JE, García AJ. Role of material-driven fibronectin fibrillogenesis in cell differentiation. Biomaterials 2011; 32(8): 20992105.

32. Ye Z, Zhang H, Luo H, Wang S, Zhou Q, DU X, et al. Temperature and pH effects on biophysical and morphological properties of self-assembling peptide RADA16-I. J Pept Sci 2008;14:152-162.

33. Keselowsky BG, Collard DM, García AJ. Surface chemistry modulates focal adhesion composition and signaling through changes in integrin binding. Biomaterials 2004; 25: 5947-5954.

34. Scotchford CA, Gilmore CP, Cooper E, Leggett GJ, Downes S. Protein adsorption and human osteoblast-like cell attachment and growth on alkylthiol on gold self-assembled monolayers, J Biomed Mater Res 2002; 59: 84-99.

35. Coelho NM, González-García C, Planell JA, Salmerón-Sánchez M, Altankov G. Different assembly of type IV collagen on hydrophilic and hydrophobic substrata alters endothelial cells interaction. Eur Cell Mater 2010; 19: 262-72. 
36. Briz N, Antolinos-Turpin CM, Alió J, Garagorri N, Gómez Ribelles JL, Gómez-Tejedor JA. Fibronectin fixation on poly(ethyl acrylate)-based copolymers. J Biomed Mater Res B Appl Biomater 2013; 101(6): 991-997.

37. Owens DK, Wendt RC. Estimation of the Surface Free Energy of Polymers. J Appl Polym Sci 1969; 13(8): 1741-1747.

38. Soria JM, Martínez Ramos C, Bahamonde O, García Cruz DM, Salmerón Sánchez M, García Esparza MA, Casas C, Guzmán M, Navarro X, Gómez Ribelles JL, García Verdugo JM, Monleón Pradas M, Barcia JA. Influence of the substrate's hydrophilicity on the in vitro Schwann cells viability. J Biomed Mater Res A 2007; 83(2): 463-70.

39. Van Krevelen DW. Properties of polymers. Chapter 13 Mechanical properties of solid polymers. Elsevier, 1997. pp. 367-437. 